\title{
THE RESISTIVE WALL MODE FEEDBACK CONTROL SYSTEM ON DIII-D
}

\author{
by \\ J.T. SCOVILLE, D.H. KELLMAN, S.G.E. PRONKO, A. NEREM, \\ R. HATCHER, ${ }^{\dagger}$ D. O'NEILL, G. ROSSI, ${ }^{\dagger}$ and M. BOLHA
}

This is a preprint of a paper to be presented at the 18th IEEE/NPSS Symposium on Fusion Engineering, October 25-29, 1999, Albuquerque, New Mexico, and to be published in the Proceedings.

†Princeton Plasma Physics Laboratory

$\ddagger_{\text {Robicon }}$ Corporation

\section{Work supported by \\ the U.S. Department of Energy \\ under Contracts DE-AC03-99ER54463, and DE-AC02-76CHO3073}




\section{DISCLAIMER}

This report was prepared as an account of work sponsored by an agency of the United States Government. Neither the United States Government nor any agency thereof, nor any of their employees, make any warranty, express or implied, or assumes any legal liability or responsibility for the accuracy, completeness, or usefulness of any information, apparatus, product, or process disclosed, or represents that its use would not infringe privately owned rights. Reference herein to any specific commercial product, process, or service by trade name, trademark, manufacturer, or otherwise does not necessarily constitute or imply its endorsement, recommendation, or favoring by the United States Government or any agency thereof. The views and opinions of authors expressed herein do not necessarily state or reflect those of the United States Government or any agency thereof. 


\section{DISCLAIMER}

Portions of this document may be illegible in electronic image products. Images are produced from the best available original document. 


\title{
The Resistive Wall Mode Feedback Control System on DIII-D*
}

\author{
J.T. Scoville, ${ }^{1}$ D.H. Kellman, ${ }^{1}$ S.G.E. Pronko, ${ }^{1}$ A. Nerem, ${ }^{1}$ R. Hatcher, ${ }^{2}$ D. O'Neill, ${ }^{2}$ \\ G. Rossi, ${ }^{2}$ and M. Bolha, ${ }^{3}$ \\ $1_{\text {General Atomics, P.O. Box 85608, San Diego, California } 92186-5608}$ \\ ${ }_{2}^{2}$ Princeton Plasma Physics Laboratory, P.O. Box 451, Princeton, New Jersey 08543 \\ ${ }^{3}$ Robicon Corporation, 500 Hunt Valley Drive, New Kensington, Pennsylvania 15608
}

\begin{abstract}
One of the primary instabilities limiting the performance of the plasma in advanced tokamak operating regimes is the resistive wall mode (RWM) [1]. The most common RWM seen in the DIII-D tokamak is originated by an $n=1$ ideal external kink mode which, in the presence of a resistive wall, is converted to a slowly growing RWM. The mode causes a reduction in plasma rotation, a loss of stored energy, and sometimes leads to plasma disruption. It routinely limits the performance of a tokamak operating near reactor relevant parameter levels. A system designed to actively control the RWM has recently been installed on the DIII-D tokamak for the control of low $\mathrm{m} \mathrm{n}=1$ modes. In initial experiments, the control system has been capable of delaying the onset of RWMs in energetic discharges for several hundred milliseconds.

The feedback control system consists of detector coils connected via control software to high power current amplifiers driving the excitation coils. The three pairs of excitation coils are each driven by a current amplifier and a DC power supply. The control signal is derived from a set of six sensor coils that measure radial flux as low as one Gauss. The signals are digitally processed by realtime software in the DIII-D Plasma Control System (PCS) to create a command that is sent to the current amplifier, with a cycle time of approximately $100 \mu \mathrm{s}$. The amplifiers, designed and fabricated by Robicon Corporation to a specification developed by PPPL and GA, are bipolar devices capable of $\pm 5 \mathrm{kA}$ at $300 \mathrm{~V}$, with an operating bandwidth of approximately $800 \mathrm{~Hz}(-3 \mathrm{~dB})$.
\end{abstract}

\section{INTRODUCTION}

Confinement of a high temperature plasma in a tokamak is subject to the constraints imposed by magnetohydrodynamic (MHD) stability. As the plasma pressure is increased, an instability that causes a deformation of the plasma surface can arise. In a plasma with no resistivity, the instability is known as the global kink mode. Placement of a perfectly conducting wall near the edge of the plasma can restore stability. In reality, the vessel wall has finite conductivity that leads to significant modification of the instability, resulting in the so-called resistive wall mode (RWM) [1].

The RWM is driven by the phase difference between the plasma surface perturbation and the dissipation of inductively coupled currents in the vessel wall. The characteristic time defining mode growth and rotation is the skin time of the vessel wall. In DIII-D, the wall time constant $\tau_{\mathrm{w}}$ for toroidal mode number $\mathrm{n}=1$ is a few milliseconds. Thus, the growth rate $\gamma \leq 1 / \tau_{w}$ is relatively slow and the rotation frequency $f \sim$ $1 / 2 \pi \tau_{\mathrm{w}}$ is tens of Hertz. The mode rotation is slow enough that an active feedback system should be able to interact with the mode and possibly stabilize it.

The amplitude of a typical RWM in DIII-D is on the order of a few Gauss to a few tens of Gauss. A carefully constructed sensor coil mounted outside the vacuum vessel can detect this level of slowly varying magnetic field. In response to the detection, an excitation coil can be used to impose a comparable magnetic field. For several years on DIII-D, we have been using a system of externally mounted saddle coils (the $\mathrm{C}$-coil) to apply a radial magnetic field to reduce the inherent error field of the tokamak [2]. In recent experiments we have expanded the role of the C-coil to include its use in experiments to actively control the resistive wall mode.

\section{Closed LoOP SySTEM OVERVIEW}

Figure 1 shows a block diagram of the RWM feedback control system. The main components of the system are the detector coils, the analog compensation system, the feedback control software running on the PCS, the power supplies, and the excitation coils. Each of these elements will be described in more detail in the following sections.

Since the detector coils and the excitation coils are mounted outside the vacuum vessel, the question arises about how the conducting vessel walls will affect the system. A series of experiments was performed to quantify the $A C$ response of the vacuum vessel and the coupling between the excitation coil (C-coil) and the magnetic diagnostics, including the external saddle loop (ESL) system used to measure the RWM perturbation. The C-coil was energized with an $\mathrm{AC}$ waveform at several frequencies and the response of the detector coils was measured. A frequency response analysis was performed over the range $0-200 \mathrm{~Hz}$, yielding information about the vacuum vessel wall eigenmodes [3]. An example of the results is shown in Fig. 2, where the transfer function from one of the C-coil sections to its corresponding ESL detector coil is plotted. The corner frequency, as defined by a $3 \mathrm{~dB}$ decrease in the transfer function, is approximately $30 \mathrm{~Hz}$.

\footnotetext{
${ }^{*}$ Work supported by U.S. Department of Energy under Contracts DE-AC03-99ER54463.and DE-AC02-76CHO3073.
} 


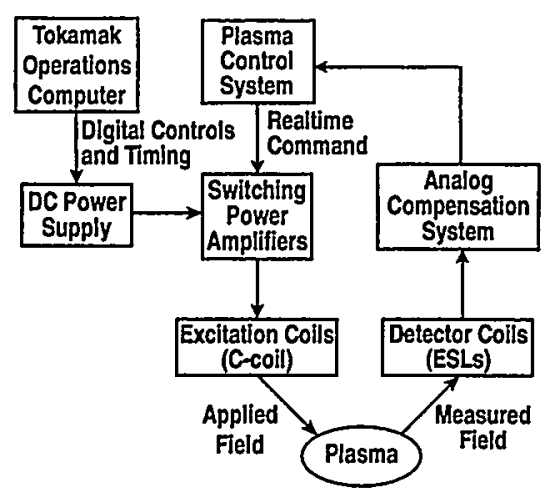

Fig. 1. Block diagram of overall RWM feedback system.

A simple two-pole fit to the data matches quite well at the lower frequencies, indicating the existence of two primary eigenmodes in the vacuum vessel, one with a time constant of $7 \mathrm{~ms}$ and another lower amplitude mode with a $1 \mathrm{~ms}$ time constant. The analytical results translate to a physical interpretation of the eigenmodes as eddy currents in the vacuum vessel wall with primarily an $n=1$ component $(7 \mathrm{~ms}$ time constant) and an $\mathrm{n}=3$ harmonic ( $1 \mathrm{~ms}$ time constant). This is to be expected since the $\mathrm{C}$-coil is configured to produce only odd toroidal mode numbers. The results of these AC response experiments are used in the modeling to help predict the system response in the presence of plasma.

\section{EXCITATION COILS}

The coil system used to apply the radial magnetic field to the edge of the plasma is the same one used routinely for error field correction on DIII-D since 1994 [4]. The C-coil system consists of six saddle coils, each $1.6 \mathrm{~m}$ high, $60^{\circ}$ wide toroidally, and spanning the tokamak at midplane (Fig. 3). Each coil consists of four turns of $750 \mathrm{MCM}$ cable, capable of carrying pulses of $5 \mathrm{kA}$ for several seconds and producing a radial field up to $30 \mathrm{G}$ at the edge of the plasma. Diametrically opposite sections are wired in antiseries to produce the primarily $n=1$ field that matches the toroidal mode number of most of the plasma instabilities (including the RWM). The inductance of one C-coil section (one pair of saddle coils) is approximately $200 \mu \mathrm{H}$ and the resistance is about $10 \mathrm{~m} \Omega$. The electrical characteristics require only a few volts for the slowly varying currents used for routine error

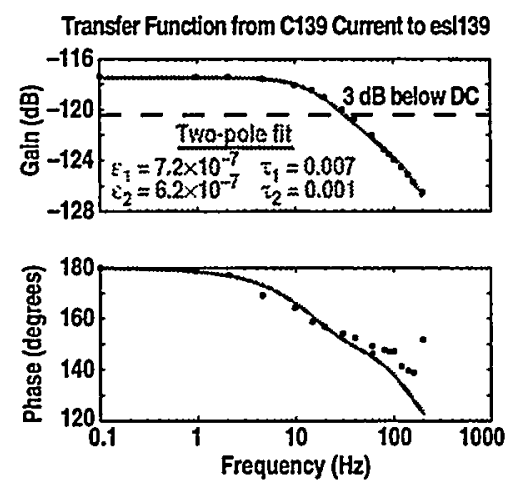

Fig. 2. Frequency response of saddle loop detector.

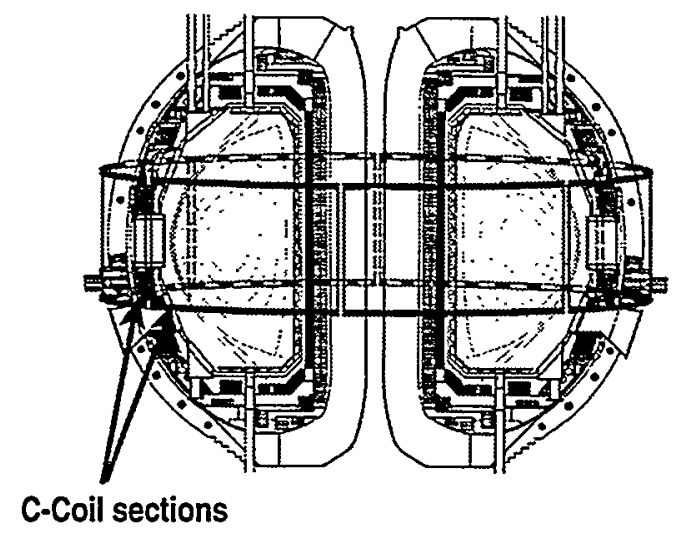

Fig. 3. Cross section of DIII-D tokamak showing C-coil.

field correction. For the RWM feedback control experiments, the voltage demands are much higher, since the system must respond quickly to commands for rapid changes in the current.

\section{DETECTOR COILS AND COMPENSATION SYSTEM}

Six new saddle coils were recently installed outside the DIII-D vacuum vessel for measurement of the radial magnetic field. The ESLs were installed as close as possible to the vessel and at the same toroidal angle and with the same toroidal extent as the $\mathrm{C}$-coil sections (Fig. 4). The one-for-one correspondence between detector and excitation coils makes the feedback system easier an 1 more efficient. The detector coils consist of a single turn of RG174 cable within 1/4" polyethylene tubing attached to existing vertical field coils. The upper toroidal leg follows the top of the F6A coil case and the lower toroidal leg follows the bottom of the F6B coil case.

During a plasma discharge, the changing flux measured by each ESL results in a voltage that is integrated and stored in the shot database. In addition, the signals from each pair of loops $180^{\circ}$ apart are subtracted electronically and integrated. These three difference signals (ESLDs) are a measure of the odd toroidal mode components of the radial magnetic field; the major component is usually $n=1$. The $n=1$ signals are connected to an analog compensation system consisting of a series of electronic summers. The outputs from these modules



Fig. 4. New external saddle loop (ESL) installed close to vessel. 
are connected to the PCS and are also digitized and stored in the shot database.

A schematic of the analog compensation system is shown in Fig. 5. The integrated ESL signals stored in the database are named for the toroidal angle of the center of the loop, e.g., ESL079. Each of the $n=1$ difference signals, e.g., (ESLD079U = ESL259 - ESL079), is also saved, before and after compensation (suffix " $U$ " indicates uncompensated). The compensation circuit has inputs from all the sources of magnetic field that may need to be removed from the measurement. The current from each of the $\mathrm{C}$-coil pairs is input to allow subtraction of the radial magnetic field $B_{r}$ induced by the C-coils. ESL coils that are not perfectly vertically aligned may measure some net vertical field $B_{z}$. Subtraction of this term is enabled by an input that is the sum of three magnetic probes located on the outboard midplane vessel wall. Carefully balancing the ESLD pairs for differences in size or orientation is allowed by connecting just one of the ESL signals (ESL079). Finally, after all the previous terms have been carefully adjusted, additional inputs from poloidal field coils very close to the ESL coils (F6A, F6B, and ECOIL) compensate for the remaining pickup. Note that the toroidal field is not compensated since the critical measurements during plasma discharges are made during the flattop phase when there is no changing toroidal field. The compensated signal is a measurement of the radial magnetic field from the plasma alone, with a relatively high signal-tonoise ratio. The "noise" level of the system is approximately $0.5 \mathrm{G}$.

\section{POWER SUPPLIES}

Each of the three C-coil pairs is driven by a current amplifier and a DC power supply. The power supplies can deliver unipolar currents up to $5 \mathrm{kA}$ and voltages up to $300 \mathrm{~V}$. These supplies (C-supplies) were previously used in voltage feedback regulation mode to produce varying currents in the

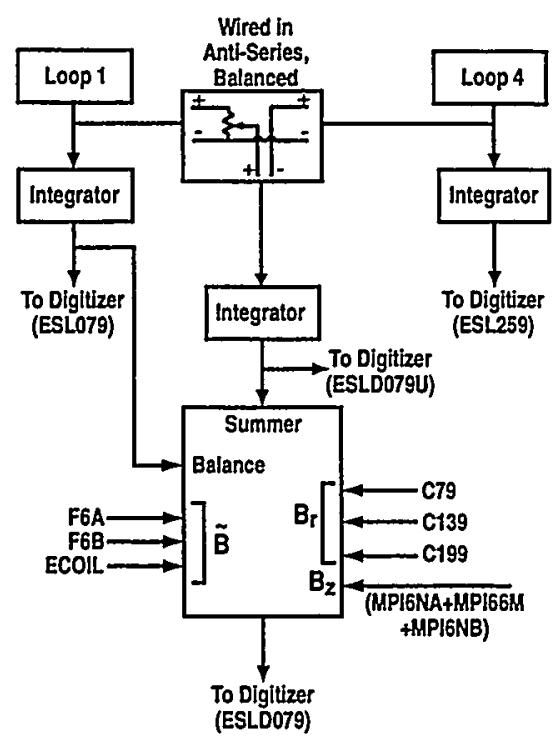

Fig. 5. ESL difference signal analog compensation system.
C-coils as required for error field correction. To meet the requirements of RWM control, a bipolar current source was needed which could regulate faster than the $\mathrm{C}$-supplies.

Princeton Plasma Physics Laboratory and General Atomics developed a specification for a switching power amplifier, which was subsequently designed and fabricated by Robicon Corp. (New Kensington, PA). This amplifier can be energized by a C-supply to meet the requirements of RWM control. The amplifier contains three input capacitor banks and three H-bridge switching power cells, each capable of producing a regulated current up to $\pm 1667 \mathrm{~A}$. Cell outputs are individually and simultaneously controllable as three separate current sources, or they may be connected in parallel for single-source output up to $\pm 5 \mathrm{kA}$. The combination of a Csupply and amplifier can be used to drive all three $\mathrm{C}$-coil pairs at moderate currents or, alternatively, to drive a single coil pair at higher current. Three amplifiers were delivered to DIII-D, and each was joined with onc C-supply to energize one $\mathrm{C}$-coil pair. The $\mathrm{C}$-supplies were reconfigured to provide fixed regulated voltage inputs (+300 VDC) to their respective amplifiers. Each supply charges the amplifier's capacitor banks and maintains input voltage as the cells regulate output current. The amplifiers were designed with a control bandwidth of $800 \mathrm{~Hz}$ to allow a fast response time. An example is shown in the step response waveform of Fig. 6 .

\section{FEEDBACK CONTROL SYSTEM}

The switching power amplifiers receive commands from the PCS to control the current in the C-coils. The real-time PCS functions are handled by a system of six parallel CSPI Inc. Intel i860 CPUs with a customized VME-based data interface [5]. The PCS cycle time is defined by the time required to read the input data, perform the feedback calculations, and output the commands. For the bulk of the control algorithms (shape control, density control, etc.) the total cycle time is approximately $1 \mathrm{~ms}$. However, one of the CPUs (CPU4) is dedicated to performing fast calculations for specific purposes such as RWM feedback control. The growth rate of the mode and the desired minimal phase shift in the control loop necessitates the use of the faster CPU4, which has a loop cycle time of approximately $100 \mu$ s for RWM feedback control.

With the recent addition of new PCS software, the Ccoils, typically used for error field control on all plasma discharges, can now be used for feedback control of the

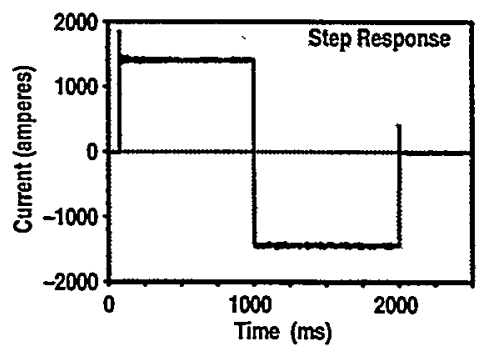

Fig. 6. Response of switching power amplifier to a square wave command. 
RWM. The error field and RWM control algorithms are mutually exclusive, requiring the operator to choose between them with a preprogrammed waveform. A flowchart illustrating the functionality of the PCS software for C-coil control is shown in Fig. 7. Error field control (left-hand side of flowchart) uses the measured currents from the known sources of error fields (the field-shaping F-coils and the toroidal field TF-coil) and generates commands to the C-coil power supplies based on the solution of a set of equations that minimize the error field. The RWM feedback code (righthand side) uses the ESLD measurements instead and generates commands using Fourier decomposition of the ESLD signals. A term proportional to cosine components $\left(\mathrm{C}_{79}\right.$, e.g.) causes current distributions in the C-coils that match the phase of the ESLD signals and a term proportional to sine components (e.g., $S_{79}$ ) imposes a phase shift for rotational drive:

$$
\begin{aligned}
& \operatorname{com}_{79}=A * C_{79}+B * S_{79} \\
& \operatorname{com}_{139}=A * C_{139}+B * S_{139} \\
& \operatorname{com}_{199}=A * C_{199}+B * S_{199}
\end{aligned}
$$

The programmable coefficients $A$ and $B$ allow comparison of the effectiveness of the "smart shell" approach (using the cosine term to cancel direct flux) and the "rotating shell" method (using the sine term to try to rotate the mode).

\section{PRELIMINARY RESULTS}

The RWM feedback control system was recently used 'vith plasma discharges for preliminary proof-of-principle experiments. An example of some initial results is shown in Fig. 8. The RWM feedback was active throughout the

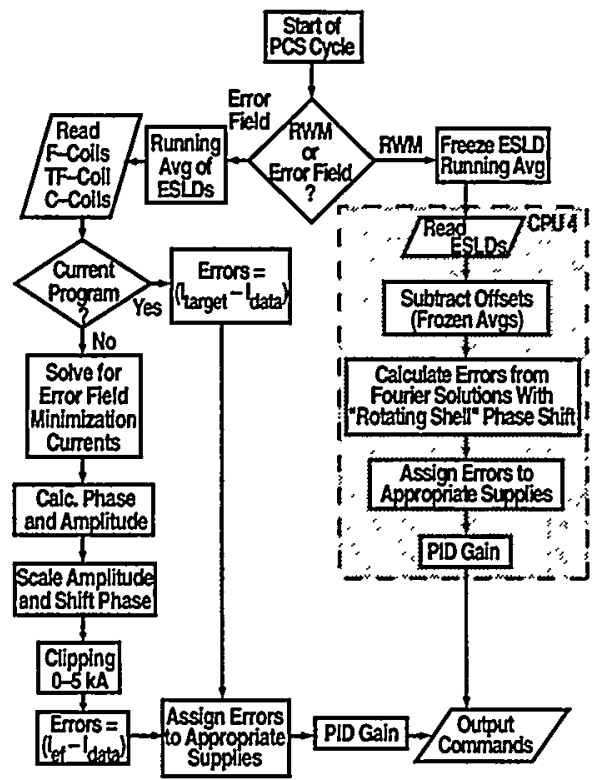

Fig. 7. Flowchart of PCS C-coil control algorithm.

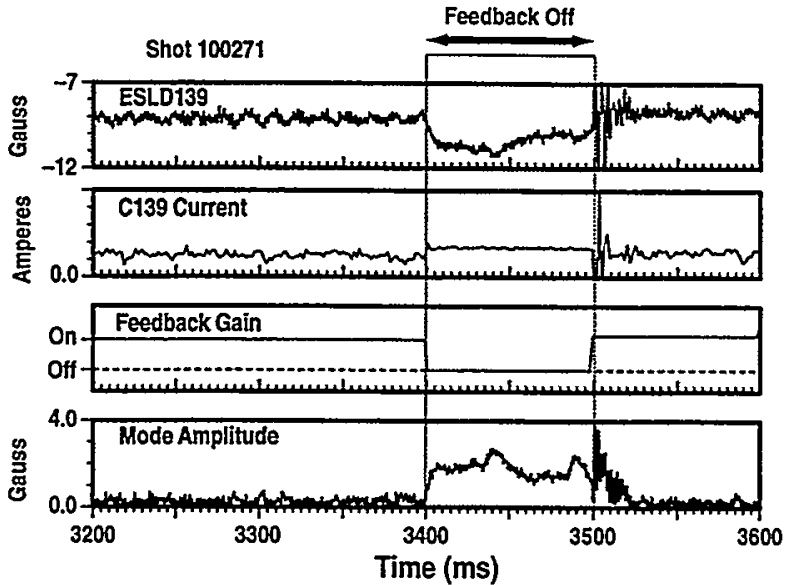

Fig. 8. A resistive wall mode appears when feedback is switched off.

discharge except for the time period $3400-3500 \mathrm{~ms}$. With the feedback switched off, the C-coil stops responding to the ESLD signal and reverts to a constant level pre-programmed for error field correction. A resistive wall mode grows up immediately to an amplitude of 2-3 G and persists until the RWM feedback is turned back on.

\section{CONCLUSIONS AND FUTURE WORK}

Three new fast, bipolar switching power amplifiers have been added to the $C$-coil power supply system previously used for routine error field correction on DIII-D. New saddle loop detector coils and new control software added to the PCS allow active closed loop feedback control of the resistive wall mode.

Future experiments will be aimed at optimizing the feedback algorithm, guided by detailed modeling of the controls, amplifier, coil system and the plasma response, to allow reliable feedback stabilization of the resistive wall mode for extended durations.

\section{REFERENCES}

[1] A.M. Garofalo, et. al., Phys Rev Lett 82 (1999) 3811.

[2] R.J. La Haye and J.T. Scoville, Rev Sci Instrum 62 (1991) 2146.

[3] J.T. Scoville, et. al., Bull Am Phys Soc 43 (1998) 1762.

[4] J.T. Scoville and R.J. La Haye, Bull Am Phys Soc 40 (1995) 1788.

[5] B. Penaflor, J.R. Ferron, M.L. Walker, Proc. 19th Symposium on Fusion Technology, September 16-20, 1996, Lisbon, Portugal, Vol. 1965 (1997). 